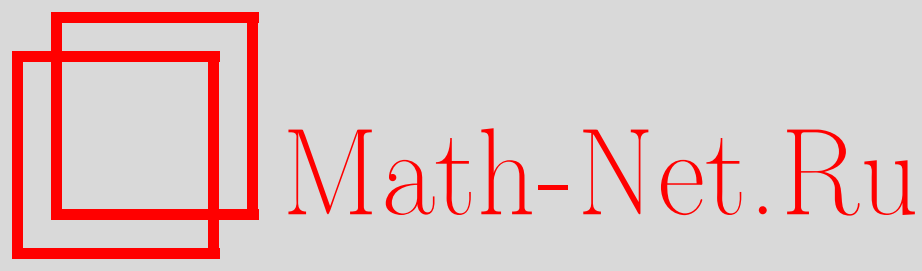

А. Е. Лобанов, Динамическое представление операторов для дираковской частицы в плосковолновом поле, $Т М \Phi$, 2015, том 182, номер 1, 112-123

DOI: https://doi.org/10.4213/tmf8743

Использование Общероссийского математического портала Math-Net.Ru подразумевает, что вы прочитали и согласны с пользовательским соглашением http://www . mathnet.ru/rus/agreement

Параметры загрузки:

IP : 54.198 .67 .100

26 апреля 2023 г., 13:56:46

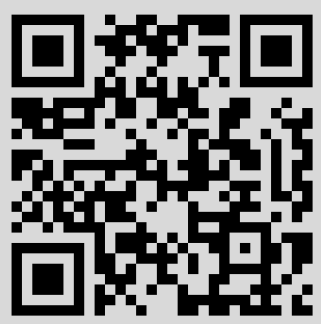




\title{
ДИНАМИЧЕСКОЕ ПРЕДСТАВЛЕНИЕ ОПЕРАТОРОВ ДЛЯ ДИРАКОВСКОЙ ЧАСТИЦЫ В ПЛОСКОВОЛНОВОМ ПОЛЕ
}

\begin{abstract}
Найден явный вид интегралов движения для дираковской частицы, находящейся в плосковолновом поле. Данные операторы представляют собой реализацию алгебры Ли группы Пуанкаре в случае, когда пространство представления состоит из решений уравнения Дирака-Паули для частицы в плосковолновом поле.
\end{abstract}

Ключевые слова: группа Пуанкаре, алгебра Ли, уравнение Дирака-Паули, интегралы движения, плосковолновое поле.

DOI: $10.4213 / \operatorname{tmf} 8743$

\section{1. ВВЕДЕНИЕ}

В математическом аппарате квантовой теории поля принято описывать элементарную частицу с помощью неприводимого унитарного представления группы Пуанкаре [1], [2]. Соответствующая алгебра Ли состоит из генераторов трансляций $P^{\mu}$, которые в квантовой теории отождествляются с импульсом частицы, и антисимметричного тензора $M^{\mu \nu}$, описывающего лоренцевские повороты, который отождествляется с тензором момента импульса.

Как хорошо известно, неприводимые представления, отвечающие массивным частицам, характеризуются значениями двух инвариантов группы:

$$
P^{2} \equiv P^{\mu} P_{\mu}=m^{2}, \quad W^{2} \equiv W^{\mu} W_{\mu}=-m^{2} s(s+1),
$$

где

$$
W^{\mu}=-\frac{1}{2} \mathrm{e}^{\mu \nu \rho \lambda} M_{\nu \rho} P_{\lambda}
$$

- вектор Паули-Любаньского-Баргмана. При этом $m^{2}$ отождествляется с квадратом массы частицы, а $s-$ с величиной спина частицы.

* Физический факультет, Московский государственный университет им. М. В. Ломоносова, Москва, Россия. Е-mail: lobanov@phys.msu.ru 
Трехмерный вектор $\mathbf{S}$ спина частицы представляет собой совокупность коэффициентов разложения вектора $W^{\mu}$ по пространственноподобным нормалям $s_{i}^{\mu}$ (здесь $i=1,2,3),\left(s_{i} P\right)=0,\left(s_{i} s_{j}\right)=-\delta_{i j}$ :

$$
S_{i}=-\frac{1}{\sqrt{P^{2}}}\left(W s_{i}\right)
$$

Разумеется, $\left[S_{i}, S_{j}\right]=i e_{i j k} S_{k}$, а выбор нормалей ничем не ограничен, т. е. можно конструировать спиновые операторы, определяющие проекцию спина на любое направление в произвольной лоренцевой системе отсчета.

Условие, выделяющее пространство унитарного представления, называется волновым уравнением для частицы, обладающей массой $m$ и спином $s[3]$ (см. также книгу [4]). Волновое уравнение для частиц со спином $s=1 / 2$ есть не что иное, как уравнение Дирака $(\hat{p}-m) \Psi(x)=0$. Здесь $\hat{p} \equiv \gamma^{\mu} p_{\mu}$, где $\gamma^{\mu}-$ матрицы Дирака.

В координатном представлении реализация генераторов группы и вектора Паули-Любаньского-Баргмана следующая:

$$
p^{\mu}=i \partial^{\mu}, \quad m^{\mu \nu}=x^{\mu} p^{\nu}-x^{\nu} p^{\mu}+\frac{i}{2} \sigma^{\mu \nu}, \quad w^{\mu}=\frac{1}{2} \gamma^{5}\left(\gamma^{\mu} \hat{p}-p^{\mu}\right),
$$

где $\sigma^{\mu \nu}=\left(\gamma^{\mu} \gamma^{\nu}-\gamma^{\nu} \gamma^{\mu}\right) / 2$. Скалярное произведение в пространстве дираковских биспиноров определено стандартным образом:

$$
\left(\Psi_{f}, \Psi_{i}\right)=\int \Psi_{f}^{\dagger}(\mathbf{x}, t) \Psi_{i}(\mathbf{x}, t) d^{3} x
$$

Операторы $p^{\mu}, m^{\mu \nu}$, а также $w^{\mu}$ коммутируют с оператором уравнения Дирака. Следовательно, их можно отождествить с наблюдаемыми физическими величинами, так как в релятивистской квантовой механике наблюдаемыми могут считаться только интегралы движения [5].

K сожалению, данное описание характеристик частицы не может быть непосредственно использовано при наличии внешних полей. Уравнение Дирака для частицы во внешнем поле имеет вид

$$
(i \hat{\partial}-e \hat{A}-m) \Psi(x)=0
$$

т. е. получается путем "удлинения производной" - добавлением потенциала внешнего поля, умноженного на заряд. Ясно, что в этом случае введенные операторы $p^{\mu}$ и $w^{\mu}$ уже не всегда являются интегралами движения. Поэтому для классификации состояний частицы во внешнем поле используются их линейные комбинации, коэффициенты которых могут явно зависеть от координат. Процедура нахождения таких комбинаций, в частности спиновых операторов, детально описана, например, в книге [6]. Однако этим операторам, особенно спиновым, как правило, нелегко дать физическую интерпретацию, что зачастую приводит к логическим трудностям. Еще большие сложности возникают при рассмотрении уравнения Дирака-Паули, получающегося путем добавления в (1.2) феноменологического члена, описывающего взаимодействие аномального магнитного момента $\mu_{0}$ с внешним полем [7]:

$$
\left(i \hat{\partial}-e \hat{A}-\frac{i}{2} \mu_{0} F^{\alpha \beta} \sigma_{\alpha \beta}-m\right) \Psi(x)=0 .
$$


В силу вышеизложенного было бы весьма желательно и для частиц, находящихся во внешних полях, дать столь же подробное и наглядное описание их характеристик, как и для свободных частиц. Возможность такого описания основана на следующих соображениях. Так как неприводимое представление определено с точностью до преобразования эквивалентности, разумно поставить задачу о нахождении такой реализации алгебры Ли группы Пуанкаре (так называемого динамического представления), для которой условие неприводимости сведется к волновому уравнению, описывающему частицу в заданном внешнем поле. Следовательно, надо найти оператор $U\left(x, x_{0}\right)$, который переводит решения волнового уравнения для свободной частицы $\left(D_{0}(x)-m\right) \Psi_{0}(x)=0$ в решения уравнения для частицы во внешнем поле

$$
(D(x)-m) \Psi(x)=0,
$$

другими словами, $U\left(x, x_{0}\right) \Psi_{0}(x)=\Psi(x)$. Таким образом, оператор $U\left(x, x_{0}\right)$ должен быть сплетающим оператором в смысле Дарбу [8]. Этот оператор должен удовлетворять уравнению

$$
D(x) U\left(x, x_{0}\right)-U\left(x, x_{0}\right) D_{0}(x)=0
$$

и дополнительному условию

$$
\lim _{x \rightarrow x_{0}} U\left(x, x_{0}\right) \Psi_{0}(x)=\Psi_{0}\left(x_{0}\right) .
$$

При этом генераторы

$$
\widetilde{P}^{\mu}=U P^{\mu} U^{-1}, \quad \widetilde{M}^{\mu \nu}=U M^{\mu \nu} U^{-1}
$$

можно интерпретировать как начальные импульс и тензор момента импульса соответственно, а вектор Паули-Любаньского-Баргмана и трехмерный вектор спина можно конструировать таким же образом, как и в случае свободной частицы. Естественно, эти операторы будут коммутировать с оператором уравнения (1.3), поскольку операторы, задающие начальные условия, по определению являются интегралами движения [9].

Ясно, что нахождение явного вида операторов $U\left(x, x_{0}\right)$ в общем случае представляет собой весьма сложную задачу. $\mathrm{K}$ настоящему времени реализовать сформулированную выше программу удалось только для уравнения, которое описывает нейтральную частицу, находящуюся в постоянном однородном электромагнитном поле с нулевым вторым инвариантом и постоянном аксиально-векторном конденсате [10]. Эта задача возникает в физике массивного нейтрино, а также в рамках обобщения Стандартной модели, которое в литературе называется Standard Model Extension [11].

Возможность найти сплетающий оператор в указанном случае обусловлена тем, что поведение частицы в такой ситуации является квазиклассическим. При этом оператор представляет собой достаточно простой объект - интегральный оператор Фурье (см., например, монографию [12]). Однако еще в работе [13] было обращено внимание на то, что так называемые решения Волкова уравнения Дирака для частицы в плосковолновом поле можно рассматривать как результат действия интегрального оператора Фурье (естественно, в указанной работе такая терминология не применялась) на решение уравнения Дирака для свободной частицы. Кроме того, в статье [14] было показано, что движение в плосковолновом поле нейтральной 
частицы, обладающей аномальным магнитным моментом, является квазиклассическим. Поэтому можно надеяться, что сплетающий оператор имеет и в этом случае достаточно простой вид. Его нахождению и построению генераторов алгебры Ли группы Пуанкаре для дираковской частицы в плосковолновом поле и посвящена настоящая статья.

\section{2. РЕШЕНИЕ ДЛЯ НЕЙТРАЛЬНОЙ ЧАСТИЦЫ}

Решения уравнения Дирака-Паули для частицы в волне линейной поляризации и в монохроматической циркулярно поляризованной волне известны давно [15]. Значительно позже в работе [16] были найдены решения для плосковолновых полей, отличающихся от указанных. Используя идеологию этой работы, найдем сплетающий оператор для уравнения Дирака-Паули.

Чтобы записать потенциал и тензор поля, введем базис нулевой плоскости в пространстве Минковского:

$$
\begin{gathered}
n^{\mu}=(1, \mathbf{n}), \quad n_{+}^{\mu}=\frac{1}{2}(1,-\mathbf{n}), \\
a_{1}^{\mu}=\left(0, \mathbf{a}_{1}\right), \quad a_{2}^{\mu}=\left(0, \mathbf{a}_{2}\right) . \\
\left(\mathbf{n a}_{i}\right)=0, \quad\left(\mathbf{a}_{1} \mathbf{a}_{2}\right)=0, \quad \mathbf{n}^{2}=\mathbf{a}_{i}^{2}=1 .
\end{gathered}
$$

Пусть волновой вектор плосковолнового поля $k^{\mu} \sim n^{\mu}$. Тогда векторы $\mathbf{a}_{1}, \mathbf{a}_{2}$ представляют собой орты поляризации.

Выберем потенциал плосковолнового поля в виде $A^{\mu}=a_{1}^{\mu} A_{1}+a_{2}^{\mu} A_{2}$. Тогда тензор плосковолнового поля определяется формулой

$$
F^{\mu \nu}=A_{1}^{\prime}\left[n^{\mu} a_{1}^{\nu}-a_{1}^{\mu} n^{\nu}\right]+A_{2}^{\prime}\left[n^{\mu} a_{2}^{\nu}-a_{2}^{\mu} n^{\nu}\right] .
$$

Здесь $A_{i} \equiv A_{i}\left(x_{-}\right)$- произвольные функции аргумента $x_{-}=(n x)$, а штрихом обозначена производная по этому аргументу: $A_{i}^{\prime}=d A_{i} / d x_{-}$.

Следовательно, уравнение Дирака-Паули можно записать так:

$$
\left(\hat{p}-i \mu_{0} \hat{n} \hat{A}^{\prime}-m\right) \Psi(x)=0, \quad p^{\mu} \equiv i \partial^{\mu} .
$$

Уравнение (1.4) для оператора $U\left(x, x_{0}\right)$ имеет вид

$$
\left(\hat{p}-i \mu_{0} \hat{n} \hat{A}^{\prime}\right) U\left(x, x_{0}\right)=U\left(x, x_{0}\right) \hat{p},
$$

причем очевидно, что $U^{-1}=\gamma^{0} U^{\dagger} \gamma^{0}$.

Домножая уравнение (2.3) справа на $\hat{p}$, получаем

$$
p^{2} U\left(x, x_{0}\right)=U\left(x, x_{0}\right) p^{2}+i \mu_{0}\left(\hat{p} \hat{n} \hat{A}^{\prime}+\hat{n} \hat{A}^{\prime} \hat{p}\right) U\left(x, x_{0}\right) .
$$

Рассмотрим частное решение $U_{(\mu)}$ этого уравнения, считая, что $U_{(\mu)}$ является функцией только переменной $x_{-}$. Тогда

$$
U_{(\mu)}^{\prime}=\frac{\mu_{0}}{2 p_{-}}\left(\hat{p} \hat{n} \hat{A}^{\prime}+\hat{n} \hat{A}^{\prime} \hat{p}\right) U_{(\mu)}
$$

Решение этого уравнения, удовлетворяющее условию (1.5), имеет вид

$$
U_{(\mu)}=T\left(p ; x_{-}, x_{0-}\right)=Y_{+} F_{-}+Y_{-} F_{+}-Z_{+} F_{-} F_{+}-Z_{-} F_{+} F_{-},
$$


где

$$
F_{ \pm}=\frac{1}{4(p n)}\left(\hat{p} \hat{n} \hat{a}_{ \pm}+\hat{n} \hat{a}_{ \pm} \hat{p}\right), \quad a_{ \pm}=a_{1} \pm i a_{2},
$$

а функции $Y_{ \pm}=Y_{ \pm}\left(x_{-}, x_{0-}\right)$ и $Z_{ \pm}=Z_{ \pm}\left(x_{-}, x_{0-}\right)$ удовлетворяют системе уравнений

$$
Y_{ \pm}^{\prime}=\mu_{0} A_{ \pm}^{\prime} Z_{\mp}, \quad Z_{ \pm}^{\prime}=-\mu_{0} A_{ \pm}^{\prime} Y_{\mp},
$$

где $A_{ \pm}=A_{1}\left(x_{-}\right) \pm i A_{2}\left(x_{-}\right)$, и соотношениям

$$
Y_{ \pm}\left(x_{0-}, x_{0-}\right)=0, \quad Z_{ \pm}\left(x_{0-}, x_{0-}\right)=1 .
$$

Таким образом, задача построения решения уравнения (2.4) сводится к решению системы (2.6).

Свойства системы уравнений данного типа и ее частные решения детально исследовались в работах [17], [18]. В наиболее интересном случае циркулярной поляризации волны, когда

$$
\begin{gathered}
A_{1}=g a_{0} \cos f, \quad A_{2}=a_{0} \sin f, \\
f \equiv f\left(x_{-}\right), \quad f_{0} \equiv f\left(x_{0_{-}}\right), \quad g= \pm 1,
\end{gathered}
$$

где $f$ - произвольная гладкая функция, решение системы (2.6) имеет вид [16]

$$
\begin{aligned}
& Y_{+}=Y_{-}^{*}=\frac{i d}{\left(1+d^{2}\right)^{1 / 2}} \mathrm{e}^{i g\left(f+f_{0}\right) / 2} \sin \psi, \\
& Z_{+}=Z_{-}^{*}=\frac{1}{\left(1+d^{2}\right)^{1 / 2}} \mathrm{e}^{i g\left(f-f_{0}\right) / 2}\left\{\left(1+d^{2}\right)^{1 / 2} \cos \psi-i g \sin \psi\right\},
\end{aligned}
$$

где $\psi=\left(1+d^{2}\right)^{1 / 2}\left(f-f_{0}\right) / 2, d=2 \mu_{0} a_{0}$.

Важный частный случай поля (2.7), когда $f=\operatorname{am}\left(\omega x_{-}, k\right)$, где $\operatorname{am}(u, k)$ - эллиптическая амплитуда, был исследован в работе [19]. Потенциал такого поля выражается через эллиптические функции Якоби $\operatorname{cn}(u, k)$ и $\operatorname{sn}(u, k)$ :

$$
A_{1}=g a_{0} \operatorname{cn}\left(\omega x_{-}, k\right), \quad A_{2}=a_{0} \operatorname{sn}\left(\omega x_{-}, k\right) .
$$

Общее решение уравнения (2.3) имеет вид

$$
U\left(x, x_{0}\right)=U_{(\mu)} U_{0},
$$

где $U_{0}\left(x, x_{0}\right) \equiv U_{0}$ - оператор, переводящий решение уравнения Дирака для свободной частицы в другое решение этого уравнения. Очевидно, такой оператор должен удовлетворять соотношениям

$$
\left[\hat{p}, U_{0}\right]=0, \quad U_{0}^{-1}=\gamma^{0} U_{0}^{\dagger} \gamma^{0}, \quad U_{0}\left(x_{0}, x_{0}\right)=1 .
$$

Следовательно, любое решение уравнения Дирака-Паули (2.2) можно представить в виде

$$
\Psi(x)=T\left(p ; x_{-}, x_{0-}\right) \Psi_{0}(x),
$$

где $\Psi_{0}(x)$ - некоторое решение уравнения для свободной частицы.

Особый интерес представляет случай, когда в качестве решений уравнения без поля выбираются плоские волны. Тогда

$$
\Psi(x)=T\left(q ; x_{-}, x_{0-}\right) \mathrm{e}^{-i(q x)}(\hat{q}+m)\left(1-\gamma^{5} \widehat{S}_{0}\right) \psi^{0},
$$

и эти решения образуют полную ортонормированную систему. 


\section{3. РЕШЕНИЕ ДЛЯ ЗАРЯЖЕННОЙ ЧАСТИЦЫ}

Рассмотрим сначала заряженную частицу без аномального момента. В этом случае уравнение (1.4) для оператора $U\left(x, x_{0}\right)$ записывается так:

$$
(\hat{p}-e \hat{A}) U\left(x, x_{0}\right)=U\left(x, x_{0}\right) \hat{p} .
$$

Частное решение этого уравнения, которое зависит только от переменной $x_{-}$, имеет вид [13]

$$
U\left(x_{-}, x_{0_{-}}\right)=\left(1+e \frac{\hat{n} \hat{A}}{2 p_{-}}\right) \exp \left\{-i \int_{x_{0_{-}}}^{x_{-}} d x_{-}\left(\frac{e(p A)}{p_{-}}-\frac{e^{2} A^{2}}{2 p_{-}}\right)\right\}
$$

Если подействовать этим оператором на плосковолновые решения уравнения Дирака для свободной частицы, то получается решение Волкова [20]. Однако решение (3.1) удовлетворяет условию (1.5), только если $A_{0} \equiv A\left(x_{0-}\right)=0$. Этого недостатка лишено решение

$$
\begin{aligned}
U_{(e)}\left(p ; x, x_{0}\right)= & \left(1+e \frac{\hat{n}\left(\hat{A}-\hat{A}_{0}\right)}{2 p_{-}}\right) \mathrm{e}^{-i e\left(\left(x-x_{0}\right) A_{0}\right)} \times \\
& \times \exp \left\{-i \int_{x_{0-}}^{x_{-}} d x_{-}\left(\frac{e\left(p\left(A-A_{0}\right)\right)}{p_{-}}-\frac{e^{2}\left(A-A_{0}\right)^{2}}{2 p_{-}}\right)\right\} .
\end{aligned}
$$

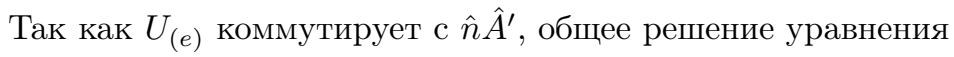

$$
\left(\hat{p}-e \hat{A}-i \mu_{0} \hat{n} \hat{A}^{\prime}\right) U\left(x, x_{0}\right)=U\left(x, x_{0}\right) \hat{p}
$$

имеет структуру

$$
U\left(x, x_{0}\right)=U_{(e)}\left(p ; x, x_{0}\right) T\left(p ; x_{-}, x_{0-}\right) U_{0}\left(x, x_{0}\right) .
$$

Подчеркнем, в этой формуле оператор $T\left(p ; x_{-}, x_{0-}\right)$ определяется тем же соотношением (2.5), что и для нейтральной частицы.

Следовательно, любое решение уравнение Дирака-Паули

$$
\left(\hat{p}-e \hat{A}-i \mu_{0} \hat{n} \hat{A}^{\prime}-m\right) \Psi=0
$$

для заряженной частицы с аномальным магнитным моментом в плосковолновом поле можно записать в виде

$$
\Psi(x)=U_{(e)}\left(p ; x, x_{0}\right) T\left(p ; x_{-}, x_{0-}\right) \Psi_{0}(x) .
$$

Когда в качестве решений уравнения без поля выбираются плоские волны, мы получаем

$$
\Psi(x)=U_{(e)}\left(q ; x, x_{0}\right) T\left(q ; x_{-}, x_{0-}\right) \mathrm{e}^{-i(q x)}(\hat{q}+m)\left(1-\gamma^{5} \widehat{S}_{0}\right) \psi^{0} .
$$

Аналогично решениям уравнения Дирака-Паули можно определить спиновую матрицу плотности. Введем оператор

$$
R\left(q ; x_{-}, x_{0-}\right)=\left(1+e \frac{\hat{n}\left(\hat{A}-\hat{A}_{0}\right)}{2 q_{-}}\right) T\left(q ; x_{-}, x_{0-}\right) .
$$


Поскольку $\gamma^{0} R^{\dagger}\left(q ; x_{-}, x_{0-}\right) \gamma^{0}=R\left(q ; x_{0-}, x_{-}\right)$, для матрицы плотности имеем

$$
\begin{aligned}
\rho(x, y)= & R\left(q ; x_{-}, x_{0-}\right) \rho_{0} R\left(q ; x_{0-}, y_{-}\right) \mathrm{e}^{-i\left(\left(q+e A_{0}\right)(x-y)\right)} \times \\
& \times \exp \left\{-i \int_{y_{-}}^{x_{-}} d x_{-}\left(\frac{e\left(q\left(A-A_{0}\right)\right)}{q_{-}}-\frac{e^{2}\left(A-A_{0}\right)^{2}}{2 q_{-}}\right)\right\},
\end{aligned}
$$

где

$$
\rho_{0}=\frac{1}{2}(\hat{q}+m)\left(1-\gamma^{5} \widehat{S}_{0}\right)
$$

- матрица плотности свободной дираковской частицы. Отметим, что в результате несложных преобразований матрицу плотности можно привести к виду

$$
\begin{aligned}
\rho(x, y)= & \frac{1}{2}\left(\hat{\pi}\left(x_{-}\right)+m\right)\left(1-\gamma^{5} \widehat{S}(x)\right) T\left(\pi\left(x_{-}\right) ; x_{-}, y_{-}\right) \times \\
& \times\left(1+e \frac{\hat{n}\left(\hat{A}\left(x_{-}\right)-\hat{A}\left(y_{-}\right)\right)}{2 q_{-}}\right) \exp \left\{-i \int_{y}^{x} d x_{\mu}\left(\pi^{\mu}\left(x_{-}\right)+e A^{\mu}\left(x_{-}\right)\right)\right\} .
\end{aligned}
$$

Входящие в эту формулы функции

$$
\pi^{\mu}\left(x_{-}\right)=q^{\mu}-e\left(A^{\mu}-A_{0}^{\mu}\right)+n^{\mu}\left[\frac{e\left(q\left(A-A_{0}\right)\right)}{q_{-}}-\frac{e^{2}\left(A-A_{0}\right)^{2}}{2 q_{-}}\right]
$$

и $S^{\mu}\left(x_{-}\right)$представляют собой решения классических уравнений Лоренца и Баргмана-Мишеля-Телегди [21] соответственно с начальными условиями $\pi^{\mu}\left(x_{0_{-}}\right)=q^{\mu}$ и $S^{\mu}\left(x_{0_{-}}\right)=S_{0}^{\mu}$, если считается, что собственное время $\tau$, как и в классической электродинамике, определено соотношением $x_{-}=\tau q_{-} / \mathrm{m}$.

Возможность представить матрицу плотности в виде (3.3) явно указывает на квазиклассичность движения частицы в плосковолновом поле.

\section{4. ДИНАМИЧЕСКОЕ ПРЕДСТАВЛЕНИЕ ОПЕРАТОРОВ}

Используем теперь результаты, полученные в предыдущих разделах, для вывода динамического представления алгебры Ли группы Пуанкаре. Рассмотрим сначала случай нейтральной частицы. Прежде всего обратим внимание на следующее обстоятельство. Пусть некоторый оператор $X$ коммутирует с оператором $D_{0}$ уравнения Дирака для свободной частицы. Тогда $X$ - некоторая функция от операторов импульса и момента импульса

$$
p^{\mu}=i \partial^{\mu}, \quad m^{\mu \nu}=\left(x^{\mu} p^{\nu}-x^{\nu} p^{\mu}\right)+\frac{1}{2} \sigma^{\mu \nu} .
$$

Поэтому оператор $\tilde{X}=U_{0} X U_{0}^{-1}$ также будет функцией (естественно, другой) от указанных операторов. Следовательно, для нахождения динамического представления нам достаточно в качестве сплетающего оператора выбрать любое частное решение уравнения (2.3). Выберем оператор $T\left(p ; x_{-}, x_{0-}\right)$, определенный формулой (2.5). Подставив этот оператор в уравнение (1.6), получаем

$$
\tilde{p}^{\mu}=p^{\mu}-i n^{\mu} \frac{\mu_{0}}{2 p_{-}}\left[\hat{p}, \hat{n} \hat{A}^{\prime}\right]_{+}
$$


Приступим теперь к нахождению спиновых операторов. Для этого удобно использовать операторы, построенные в виде сверток вектора Паули-Любаньского-Баргмана $w^{\mu}(1.1)$ с системой пространственноподобных нормалей, задающих базис плосковолнового поля:

$$
\Lambda_{1,2}^{\mu}=a_{1,2}^{\mu}-n^{\mu} \frac{\left(a_{1,2} p\right)}{p_{-}}, \quad \Lambda_{3}^{\mu}=\frac{n^{\mu}}{p_{-}}-\frac{p^{\mu}}{p^{2}} .
$$

Возникающие при этом операторы

$$
S_{\Lambda_{1,2}}=\frac{1}{2} \gamma^{5}\left(\hat{n} \frac{\left(p a_{1,2}\right)}{p_{-}}-\hat{a}_{1,2}\right), \quad S_{\Lambda_{3}}=\frac{1}{2} \gamma^{5}\left(1-\frac{\hat{n} \hat{p}}{p_{-}}\right)
$$

допускают очевидную интерпретацию. Так, оператор $S_{\Lambda_{3}}$, представляющий собой свертку вектора $w^{\mu}$ с нормалью $\Lambda_{3}^{\mu}$, определяет проекцию спина на волновой вектор плосковолнового поля в системе покоя частицы, а операторы $S_{\Lambda_{1,2}}$ характеризуют проекции спина на орты поляризации в системе покоя частицы.

Если ввести операторы $S_{\Lambda_{ \pm}}=S_{\Lambda_{1}} \pm i S_{\Lambda_{2}}$, то для операторов спина в динамическом представлении имеем

$$
\begin{aligned}
\widetilde{S}_{\Lambda_{+}} & =Z_{-}^{2} S_{\Lambda_{+}}-Y_{+}^{2} S_{\Lambda_{-}}-2 Z_{-} Y_{+} S_{\Lambda_{3}}, \\
\widetilde{S}_{\Lambda_{-}} & =Z_{+}^{2} S_{\Lambda_{-}}-Y_{-}^{2} S_{\Lambda_{+}}-2 Z_{+} Y_{-} S_{\Lambda_{3}}, \\
\widetilde{S}_{\Lambda_{3}} & =Z_{-} Y_{-} S_{\Lambda_{+}}+Z_{+} Y_{+} S_{\Lambda_{-}}+\left(Z_{+} Z_{-}-Y_{+} Y_{-}\right) S_{\Lambda_{3}},
\end{aligned}
$$

где $Z_{ \pm}, Y_{ \pm}-$решения системы (2.6).

Выпишем явный вид этих операторов для плоской волны циркулярной поляризации (см. соотношения (2.7), (2.8)), когда решение системы (2.6) известно:

$$
\begin{aligned}
\widetilde{S}_{\Lambda_{+}}= & \frac{1}{1+d^{2}} \mathrm{e}^{i g f_{0}}\left\{\mathrm{e}^{-i g f}\left(\left(1+d^{2}\right)^{1 / 2} \cos \psi+i g \sin \psi\right)^{2} S_{\Lambda_{+}}+\right. \\
& \left.+\mathrm{e}^{i g f}(d \sin \psi)^{2} S_{\Lambda_{-}}-2 i d \sin \psi\left(\left(1+d^{2}\right)^{1 / 2} \cos \psi+i g \sin \psi\right) S_{\Lambda_{3}}\right\} \\
\widetilde{S}_{\Lambda_{-}}= & \frac{1}{1+d^{2}} \mathrm{e}^{-i g f_{0}}\left\{\mathrm{e}^{i g f}\left(\left(1+d^{2}\right)^{1 / 2} \cos \psi-i g \sin \psi\right)^{2} S_{\Lambda_{-}}+\right. \\
& \left.+\mathrm{e}^{-i g f}(d \sin \psi)^{2} S_{\Lambda_{+}}+2 i d \sin \psi\left(\left(1+d^{2}\right)^{1 / 2} \cos \psi-i g \sin \psi\right) S_{\Lambda_{3}}\right\} \\
\widetilde{S}_{\Lambda_{3}}= & \frac{1}{1+d^{2}}\left\{i d \mathrm{e}^{i g f} \sin \psi\left(\left(1+d^{2}\right)^{1 / 2} \cos \psi-i g \sin \psi\right) S_{\Lambda_{-}}-\right. \\
& -i d \mathrm{e}^{-i g f} \sin \psi\left(\left(1+d^{2}\right)^{1 / 2} \cos \psi+i g \sin \psi\right) S_{\Lambda_{+}}+ \\
& \left.+\left(1+d^{2}\left(\cos ^{2} \psi-\sin ^{2} \psi\right)\right) S_{\Lambda_{3}}\right\}
\end{aligned}
$$

Характерной особенностью полученных спиновых операторов является явная зависимость от $x_{0-}$. При этом операторы (4.3) выделяют такие состояния, в которых поляризация частицы при $x_{-}=x_{0-}$ описывается операторами $S_{\Lambda_{i}}$.

Построим теперь спиновые операторы, в которых зависимость от начальной поляризации отсутствует. Очевидно, что такие операторы будут являться линейной 
комбинацией операторов (4.4) с коэффициентами, зависящими только от $x_{0-}$. В результате несложных вычислений получаем

$$
\begin{aligned}
S_{ \pm}^{\mathrm{c}}= & \frac{1}{\left(1+d^{2}\right)^{1 / 2}} \mathrm{e}^{ \pm i g\left(1+d^{2}\right)^{1 / 2} f_{0}}\left\{-g d \widetilde{S}_{\Lambda_{3}}+\right. \\
& \left.+\frac{1}{2}\left(1 \pm\left(1+d^{2}\right)^{1 / 2}\right) \mathrm{e}^{-i g f_{0}} \widetilde{S}_{\Lambda_{+}}+\frac{1}{2}\left(1 \mp\left(1+d^{2}\right)^{1 / 2}\right) \mathrm{e}^{i g f_{0}} \widetilde{S}_{\Lambda_{-}}\right\} \\
S_{3}^{\mathrm{c}}= & \frac{1}{\left(1+d^{2}\right)^{1 / 2}}\left\{\widetilde{S}_{\Lambda_{3}}+\frac{g d}{2}\left(\mathrm{e}^{-i g f_{0}} \widetilde{S}_{\Lambda_{+}}+\mathrm{e}^{i g f_{0}} \widetilde{S}_{\Lambda_{-}}\right)\right\} .
\end{aligned}
$$

Чтобы выразить эти операторы через операторы (4.2), достаточно в формулах (4.5) произвести замены $\widetilde{S}_{\Lambda} \rightarrow S_{\Lambda}, f_{0} \rightarrow f$. Поэтому, в частности,

$$
S_{3}^{\mathrm{c}}=\frac{\gamma^{5}}{2\left(1-\left(2 \mu_{0} A\right)^{2}\right)^{1 / 2}}\left\{1-\frac{\hat{n} \hat{p}}{p_{-}}+2 \mu_{0}\left(\hat{n} \frac{(p A)}{p_{-}}-\hat{A}\right)\right\} .
$$

Если в формулах (4.4) провести усреднение по переменной $x_{0}$, т. е. усреднение по начальной поляризации частицы, то нетрудно убедиться, что

$$
\left\langle\widetilde{S}_{\Lambda_{3}}\right\rangle_{x_{0}}=a S_{3}^{\mathrm{c}}, \quad\left\langle\mathrm{e}^{\mp i x_{0}} \widetilde{S}_{\Lambda_{ \pm}}\right\rangle_{x_{0}}=b S_{3}^{\mathrm{c}}, \quad\left\langle\widetilde{S}_{\Lambda_{ \pm}}\right\rangle_{x_{0}}=0
$$

где $a$ и $b$ - константы, не равные нулю. Следовательно, оператор $S_{3}^{\mathrm{c}}$ выделен. Собственные волновые функции этого оператора описывают частицу, спин которой жестко привязан к внешнему полю. А именно, в системе покоя частицы ее вектор спина прецессирует с частотой волны вокруг волнового вектора, находясь в плоскости, в которой лежат волновой вектор и вектор напряженности магнитного поля. При этом угол между вектором спина и волновым вектором равен арктангенсу параметра $d$.

Тот факт, что возможно сконструировать спиновые операторы - интегралы движения, не зависящие от начальных условий, - крайне интересен. Естественно, что описанный характер прецессии есть не более чем квазиклассический образ. Однако при квазиклассическом рассмотрении процесса излучения фотонов в поле волны циркулярной поляризации нейтральной частицей с аномальным магнитным моментом было выяснено [22], что эффект радиационной самополяризации приводит к стационарной прецессии описанного выше типа, причем самополяризация полная.

Это обстоятельство является серьезным указанием на то, что при последовательном квантовом рассмотрении процесса излучения можно ожидать, что оператор $S_{3}^{\text {c }}$ описывает состояние полной самополяризации. $\mathrm{K}$ сожалению, квантовый расчет процесса излучения до сих пор не выполнен из-за серьезных технических трудностей. Тем не менее для этого предположения есть серьезные основания. При исследовании процесса излучения фотонов частицей в аксиально-векторном конденсате [23], [24] оказалось, что в процессе излучения возникает состояние с полной поляризацией. И, как было показано позднее [10], описывается такое состояние единственно возможным спиновым оператором, среднее значение которого не обращается в нуль. И, конечно же, тот же результат следует из квазиклассического рассмотрения [25]. 
Перейдем к исследованию заряженных частиц, обладающих аномальным магнитным моментом. Используя оператор $U_{(e)}\left(p ; x, x_{0}\right)$ (см. формулу $\left.(3.2)\right)$, получаем

$$
\begin{aligned}
\tilde{p}^{\mu}= & p^{\mu}-e A_{0}^{\mu}-n^{\mu}\left[\frac{e\left(p\left(A-A_{0}\right)\right)}{p_{-}}-\frac{e^{2}\left(A^{2}-A_{0}^{2}\right)}{2 p_{-}}\right]- \\
& -\frac{i n^{\mu}}{2 p_{-}}\left(e \hat{n} \hat{A}^{\prime}+\mu_{0}\left[(\hat{p}-e \hat{A}), \hat{n} \hat{A}^{\prime}\right]_{+}\right) .
\end{aligned}
$$

Спиновые операторы в этом случае определяются формулой (4.3), в которой необходимо произвести замену $p^{\mu} \rightarrow \pi^{\mu}=p^{\mu}-e A^{\mu}$. В частности, для частицы без аномального момента общий спиновый интеграл движения, давно полученный из чисто алгебраических соображений [26], на решениях уравнения Дирака совпадает с оператором

$$
Y=\left(\mathbf{l} \mathbf{a}_{1}\right) S_{\Lambda_{1}}(\pi)+\left(\mathbf{l} \mathbf{a}_{2}\right) S_{\Lambda_{2}}(\pi)+(\mathbf{l n}) S_{\Lambda_{3}}(\pi),
$$

где $\mathbf{l}$ - произвольный постоянный единичный вектор.

Еще раз подчеркнем, что собственные функции построенных нами спиновых операторов описывают не частицу, спин которой имеет фиксированную ориентацию, а частицу, спин которой совершает регулярную прецессию. Разумеется, возможно только косвенное измерение наблюдаемых такого типа. Например, поскольку характеристики излучения частицы зависят от ее спинового состояния, то исходя из анализа излучения пучка частиц можно оценить степень его поляризации и проследить динамику процесса самополяризации (см. работу [27]).

Несложно восстановить и явный вид углового момента (1.1) в динамическом представлении. Под действием оператора $T\left(p ; x_{-}, x_{0-}\right)$

$$
\begin{aligned}
\gamma^{5} & \rightarrow 4 \gamma^{5} S_{\Lambda_{3}} \widetilde{S}_{\Lambda_{3}}, \\
\gamma^{\mu} & \rightarrow \gamma^{\mu}+2 \gamma^{5} S_{\Lambda_{3}}\left[\Lambda_{-}^{\mu}\left(\widetilde{S}_{\Lambda_{+}}-S_{\Lambda_{+}}\right)-\Lambda_{+}^{\mu}\left(\widetilde{S}_{\Lambda_{-}}-S_{\Lambda_{-}}\right)\right] \\
x^{\mu} & \rightarrow x^{\mu}-i \gamma^{5} \hat{n} S_{\Lambda_{3}}\left[\Lambda_{-}^{\mu}\left(\widetilde{S}_{\Lambda_{+}}-S_{\Lambda_{+}}\right)-\Lambda_{+}^{\mu}\left(\widetilde{S}_{\Lambda_{-}}-S_{\Lambda_{-}}\right)\right] .
\end{aligned}
$$

Под действием оператора $U_{(e)}\left(p ; x, x_{0}\right)$

$$
\begin{aligned}
\gamma^{5} & \rightarrow \gamma^{5} \\
\gamma^{\mu} & \rightarrow \gamma^{\mu}+\frac{e}{p_{-}}\left[\left(A^{\mu}-A_{0}^{\mu}\right) \hat{n}-n^{\mu}\left(\hat{A}-\hat{A}_{0}\right)\right]-n^{\mu} \hat{n} \frac{e^{2}\left(A-A_{0}\right)^{2}}{2 p_{-}^{2}}, \\
x^{\mu} \rightarrow & x^{\mu}+\frac{1}{p_{-}}\left\{-i n^{\mu} \frac{e \hat{n}\left(\hat{A}-\hat{A}_{0}\right)}{2 p_{-}}+\right. \\
& \left.+\int_{x_{0-}}^{x_{-}} d x_{-}\left[e\left(A^{\mu}-A_{0}^{\mu}\right)-n^{\mu} \frac{e\left(p\left(A-A_{0}\right)\right)}{p_{-}}+n^{\mu} \frac{e^{2}\left(A^{2}-A_{0}^{2}\right)}{2 p_{-}}\right]\right\} .
\end{aligned}
$$

Формулы (4.1), (4.6)-(4.8) позволяют найти тензор углового момента. Окончательные выражения для операторов углового момента в динамическом представлении весьма громоздки, поэтому здесь не приводятся.

Таким образом, задача о нахождении динамического представления генераторов алгебры Ли для частицы в плосковолновом поле решена. 
В заключение отметим, что если мы знаем сплетающий оператор, то можем найти и оператор кинетического импульса. Оператор $U\left(x, x_{0}\right)$ перехода к динамическому представлению нормирован условием (1.5). Поэтому оператор

$$
\mathfrak{p}^{\mu}\left(x_{0}\right)=\left.U\left(x, x_{0}\right) p^{\mu} U^{-1}\left(x, x_{0}\right)\right|_{x=x_{0}}
$$

следует интерпретировать как генератор сдвига в точке $x_{0}$. Так как эта точка произвольна, оператор

$$
\mathfrak{p}^{\mu}(x)=p^{\mu}-e A^{\mu}-\frac{i n^{\mu}}{2 p_{-}}\left(e \hat{n} \hat{A}^{\prime}+\mu_{0}\left[(\hat{p}-e \hat{A}), \hat{n} \hat{A}^{\prime}\right]_{+}\right)
$$

разумно трактовать как оператор кинетического импульса. Дополнительным аргументом в пользу такой трактовки служит то, что

$$
\hat{\mathfrak{p}}=\hat{p}-e \hat{A}-i \mu_{0} \hat{n} \hat{A}^{\prime}
$$

а оператор $\mathfrak{p}^{2}$ совпадает с оператором квадрированного уравнения Дирака-Паули.

Благодарности. Автор благодарен В.Г. Багрову и А.В. Борисову за плодотворные дискуссии.

\section{Список литературы}

[1] E. P. Wigner, Ann. Math. (2), 40:1 (1939), 149-204.

[2] Н. Н. Боголюбов, А. А. Логунов, А. И. Оксак, И. Т. Тодоров, Общие приниипы квантовой теории поля, Наука, М., 1987.

[3] И. М. Гельфанд, А. М. Яглом, ЖЭТФ, 18:8 (1948), 703-733.

[4] А. Барут, Р. Рончка, Теория представлений групп и ее приложения, Мир, М., 1980.

[5] L. D. Landau, R. Peierls, Z. Phys., 69:1-2 (1931), 56-69.

[6] V. G. Bagrov, D. M. Gitman, Exact Solutions of Relativistic Wave Equations, Kluwer, Dordrecht, 1990.

[7] W. Pauli, Rev. Modern Phys., 13:3 (1941), 203-232.

[8] G. Darboux, C. R. Acad. Sci., 94 (1882), 1456-1459.

[9] И.А. Малкин, В.И. Манько, Динамические симметрии и когерентные состояния квантовых систем, Наука, М., 1979.

[10] E. V. Arbuzova, A.E. Lobanov, E. M. Murchikova, Phys. Rev. D, 81:4 (2010), 045001, 16 pp., arXiv: 0903.3358.

[11] S. M. Carroll, G. B. Field, R. Jackiw, Phys. Rev. D, 41:4 (1990), 1231-1240; D. Colladay, V. A. Kostelecký, Phys. Rev. D, 55:11 (1997), 6760-6774, arXiv: hep-ph/9703464; D. Colladay, V.A. Kostelecký, Phys. Rev. D, 58:11 (1998), 116002, 23 pp., arXiv: hep-ph/9809521; S. Coleman, S. L. Glashow, Phys. Rev. D, 59:11 (1999), 116008, 14 pp., arXiv: hep-ph/9812418.

[12] Ф. Трев, Введение в теорию псевдодифференциалъных операторов и интегральных операторов Фуръе, Мир, М., 1984.

[13] A. Chakrabarti, Nuovo Cimento A, 56:3 (1968), 604-625.

[14] А.Е. Лобанов, О. С. Павлова, Вестник МГУ. Физика, астр., 40:4 (1999), 3-5.

[15] И. М. Тернов, В. Г. Багров, В.А. Бордовицын, Ю.А. Маркин, ЖЭТФ, 52:6 (1967), $1584-1591$. 
[16] А.Е. Лобанов, Ковариантное описание спина электрона в электромагнитном поле, Препринт № 24, Изд-во МГУ, М., 1988.

[17] А. Е. Лобанов, Вестник МГУ. Физика, астр., 38:2 (1997), 59-60.

[18] V. G. Bagrov, D. M. Gitman, M. C. Baldiotti, A. D. Levin, Ann. Phys., 14:11-12 (2006), 764-789.

[19] А.С. Вшивцев, В.Ч. Жуковский, А.Е. Лобанов, Р. А. Потапов, ЯФ, 57:4 (1994), $758-763$.

[20] D. M. Wolkow, Z. Phys., 94:3-4 (1935), 250-260; Д. М. Волков, ЖЭТФ, 7:11 (1937), $1286-1289$

[21] V. Bargmann, L. Michel, V. L. Telegdi, Phys. Rev. Lett., 2:10 (1959), 435-436.

[22] A. E. Lobanov, J. Phys. A, 39:23 (2006), 7517-7529, arXiv: hep-ph/0311021.

[23] A. E. Lobanov, Phys. Lett. B, 619:1-2 (2005), 136-144, arXiv: hep-ph/0506007.

[24] V.Ch. Zhukovsky, A.E. Lobanov, E. M. Murchikova, Phys. Rev. D, 73:6 (2006), 065016, 8 pp., arXiv: hep-ph/0510391.

[25] A. E. Lobanov, A. I. Studenikin, Phys. Lett. B, 601:3-4 (2004), 171-175, arXiv: astro-ph/0408026.

[26] I. M. Ternov, V. G. Bagrov, A. M. Khapaev, Ann. Phys., 477:1 (1968), 25-32.

[27] В. А. Бордовицын, И. М. Тернов, В. Г. Багров, УФН, 165:9 (1995), 1083-1094. 